\title{
A feasibility investigation of speciation by Fe K-edge XANES using a laboratory X-ray absorption spectrometer
}

\author{
Lucy M. MOTTRAM, Samuel CAFFERKEY, Amber R. MASON, Tom OULTON, Shi Kuan SUN, \\ Daniel J. BAILEY, Martin C. STENNETT, Neil C. HYATT*
}

\author{
Department of Materials Science and Engineering, The University of Sheffield, Mappin Street, Sheffield, S1 3JD, United Kingdom; \\ n.c.hyatt@sheffield.ac.uk \\ * Corresponding author
}

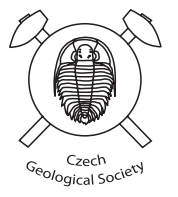

We demonstrate effective speciation of Fe in model compounds from analysis of the weak pre-edge features in $\mathrm{Fe}$ K-edge XANES spectra, with a commercially available laboratory X-ray spectrometer, using a spherically bent crystal analyser and a low-power X-ray tube, in Rowland circle geometry. Direct comparison with XANES data acquired from a third generation synchrotron bending magnet beamline, using the same specimens, validated quantitative agreement in determination of the total integrated intensity and centroid position of the pre-edge feature, which are a probe of the electronic configuration and symmetry of the absorber atom, and hence oxidation state and co-ordination number. This work opens the door to rapid and routine speciation studies of Fe by laboratory XANES, even for materials with relatively dilute absorber concentration of only a few mol. \%.

Keywords: XANES, X-ray Absorption Spectroscopy, iron, speciation, laboratory Received: 18 October 2019; accepted: 6 March 2020; handling editor: J. Plášil

\section{Introduction}

The redox chemistry of iron is of fundamental importance in Earth science and plays a crucial role in geochemical and biogeochemical processes (Taylor and Konhauser 2011). Iron is so far known to adopt formal oxidation states from $\mathrm{Fe}^{2+}$ to $\mathrm{Fe}^{6+}$, although the most common are ferrous $\mathrm{Fe}^{2+}$ and ferric $\mathrm{Fe}^{3+}$ (Greenwood and Earnshaw 1997). Redox transformations involving $\mathrm{Fe}^{2+}$ and $\mathrm{Fe}^{3+}$ are of critical importance in geological, environmental, biological and material processes, and, for example, the $\mathrm{Fe}^{2+} / \mathrm{Fe}^{3+}$ ratio is commonly exploited to constrain the conditions of mineral and glass formation (Wood and Virgo 1989; Taylor and Konhauser 2011; Joseph et al. 2017). X-ray absorption spectroscopy (XAS) has proven a powerful tool for the determination of Fe speciation in minerals and materials (Waychunas et al. 1983; Bajt et al. 1994; Galoisy et al. 2001; Petit et al. 2001; Wilke et al. 2001; Berry et al. 2003; Joseph et al. 2017); however, routine application is inhibited by the requirement to access XAS beamlines at synchrotron radiation facilities, which is highly competitive, time limited, and not immediate. The weak pre-edge features in the Fe K-edge X-ray Absorption Near Edge Structure (XANES), located c. $15-20 \mathrm{eV}$ below the edge step, have been shown to be a particularly sensitive probe of Fe speciation (Waychunas et al. 1983; Bajt et al. 1994; Galoisy et al. 2001; Petit et al. 2001; Wilke et al. 2001; Berry et al. 2003; Joseph et al. 2017). These features are associated with $1 \mathrm{~s}-3 \mathrm{~d}$ (quad- rupole) and/or 1s-4p (dipole) electronic transitions in the $\mathrm{Fe}$ absorber atom and, therefore, are sensitive to both the electronic configuration and symmetry, from which oxidation state and co-ordination number are inferred.

Wilke et al. (2001) and Petit et al. (2001), with coworkers, developed a systematic methodology to extract the normalised pre-edge features from Fe K-edge XANES data and determination of the centroid energy position and total integrated intensity, which is effectively correlated with Fe oxidation state and co-ordination number. This methodology utilised high resolution XANES data from mineral reference compounds, primarily acquired using the undulator beamline ID26 with a Si (220) double crystal monochromator, at the European Synchrotron Radiation Facility. This methodology has subsequently been widely applied (685 citations up to the end of 2019 - Wilke et al. 2001).

In recent years, a renaissance in laboratory instrumentation for X-ray absorption and emission spectroscopy has been driven by exploitation of Spherically Bent Crystal Analysers (SBCAs) in a Rowland circle geometry or bent cylindrical monochromators in von Hamos geometry (Seidler et al. 2014, 2016; Schlesiger et al. 2015; Mortensen et al. 2016; Nemeth et al. 2016; Holden et al. 2017; Malzer et al. 2018; Blachucki et al. 2019; Honkanen et al. 2019; Jahrman et al. 2019; Zeeshan et al. 2019; Mottram et al. 2020). In principle, such instrumentation may allow good quality X-ray absorption or emission spectroscopy to be routinely acquired from 


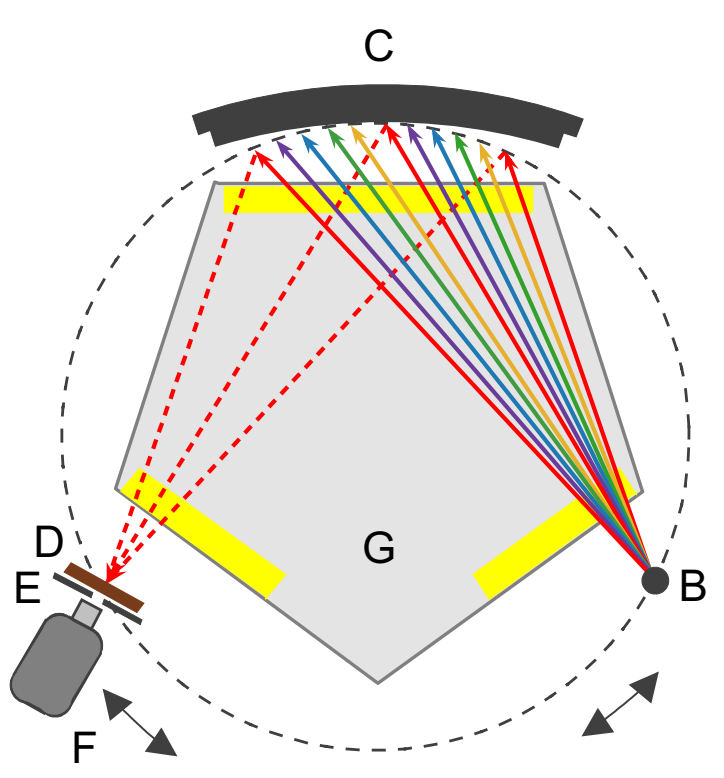

A

Fig. 1 Schematic representation of Rowland circle geometry for laboratory transmission X-ray Absorption Spectroscopy, as described in the text, showing: Rowland circle (A), broadband low power X-ray source (B), spherically bent crystal analyser (C), sample (D) and slit (E) arrangement, silicon drift detector (F) and helium-filled beam path with kapton windows $(\mathrm{G})$.

materials with moderately dilute to concentrated absorbers in a matter of hours, without recourse to a synchrotron light source. Here, we report the results of a preliminary comparative investigation of $\mathrm{Fe}$ speciation by analysis of weak pre-edge features in Fe K-edge XANES using a commercially available laboratory spectrometer based on the design of Seidler et al. (2014), which is also capable of acquisition of the full Extended X-ray Absorption Fine

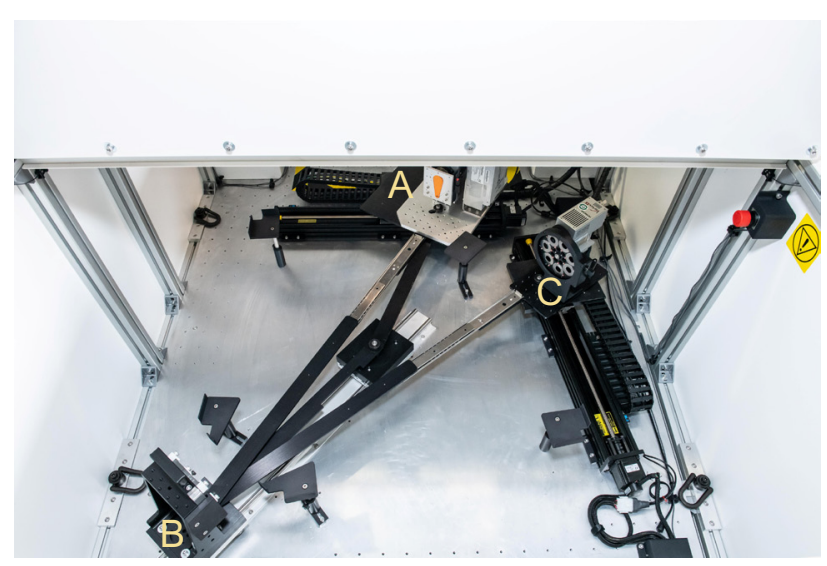

Fig. 2 Photograph of EasyXAFS XES100 spectrometer arrangement shown in Fig. 1, with helium-filled beam-path removed, to show linear translation stages and steering bars used to maintain alignment of $\mathrm{X}$-ray source (A), spherically bent crystal analyser (B) as well as sample, slit and detector assembly $(\mathrm{C})$.
Structure (EXAFS) in favourable circumstances (see also, for further developments: Seidler et al. 2016; Mortensen et al. 2016; Jahrman et al. 2019). The spectrometer arrangement utilises SBCAs in a $1 \mathrm{~m}$ Rowland circle geometry (Figs 1 and 2), in which energy scanning is achieved through symmetrical movement of detector and source. Linear translation stages are used to increment Bragg angle steps, with the alignment of source, SBCA and detector maintained by steering bars. The spectrometer operates close to back-scattering geometry to maximise resolution and thus requires a different SBCA to scan several hundred $\mathrm{eV}$. In our set up, the high efficiency of the SBCAs enables the use of a relatively inexpensive, low-power, air-cooled, X-ray source $(100 \mathrm{~W})$, which is sufficient for transmission mode investigation of moderately dilute absorbers in a favourable matrix, with a data collection time of several hours (it is also possible to utilise a fixed X-ray source of $1.5 \mathrm{~kW}$ with suitable modification of the optical arrangement, as highlighted by Honkanen et al. 2019). With robust initial SBCA alignment (Mortensen and Seidler 2017), change over and alignment of SBCAs is straightforward and can be achieved in 20 minutes or less. In the following, we first compare the resolution performance of this laboratory spectrometer in comparison with a synchrotron bending magnet beamline at a third generation synchrotron source. Using the well-established methodology (Petit et al. 2001; Wilke et al. 2001), we then demonstrate that laboratory XANES data from reference compounds can be analysed to accurately infer Fe speciation from the weak pre-edge features, even in the case of a relatively low Fe concentration of a few mol. \%.

\section{Materials and methods}

\subsection{Materials}

The materials investigated in this study are summarised in Tab. $1 . \mathrm{NaFeSi}_{2} \mathrm{O}_{6}$ and $\mathrm{FePO}_{4}$ are characterised by $\mathrm{Fe}^{3+}$, co-ordinated by oxygen in an octahedral and tetrahedral coordinations, respectively (Arnold 1986; Baum et al. 1988). $\mathrm{Fe}_{2} \mathrm{SiO}_{4}$ and $\mathrm{Fe}_{1.5} \mathrm{Mg}_{0.5} \mathrm{Al}_{9.1} \mathrm{Si}_{3.9}(\mathrm{OH})_{2}$ are characterised by $\mathrm{Fe}^{2+}$, coordinated by oxygen in an octahedral and tetrahedral coordinations, respectively (Smyth 1975; Hawthorne et al. 1993). Polycrystalline $\mathrm{FePO}_{4}$ and $\mathrm{Fe}_{2} \mathrm{SiO}_{4}$ were, respectively, synthesised by: (1) solid-state reaction of $\mathrm{Fe}_{2} \mathrm{O}_{3}$ and $\mathrm{NH}_{4} \mathrm{H}_{2} \mathrm{PO}_{4}$ at $900^{\circ} \mathrm{C}$ in air for $16 \mathrm{~h}$ and (2) solid-state reaction of stoichiometric quantities of $\mathrm{Fe}, \mathrm{Fe}_{2} \mathrm{O}_{3}$ and $\mathrm{SiO}_{2}$ at $750^{\circ} \mathrm{C}$ for $48 \mathrm{~h}$ in a sealed, evacuated, quartz tube. Mineral specimens of aegirine $-\mathrm{NaFeSi}_{2} \mathrm{O}_{6}$ (Langesund Fjord, Norway), and staurolite $-\mathrm{Fe}_{1.5} \mathrm{Mg}_{0.5} \mathrm{Al}_{9.1} \mathrm{Si}_{3.9}(\mathrm{OH})_{2}$ (Georgia, USA), were provided from our own collection. All materials 
Tab. 1 Materials analysed in this study, summarising Fe oxidation state, co-ordination number (CN) by oxygen (and symmetry), Fe concentration, and data collection time

\begin{tabular}{|c|c|c|c|c|}
\hline Material & Fe oxidation state & $\mathrm{CN}$ & Fe concentration (mol. \%) & Laboratory data collection time (h) \\
\hline $\begin{array}{l}\mathrm{NaFeSi}_{2} \mathrm{O}_{6} \\
\text { Aegirine: Langesund Fjord (Norway) }\end{array}$ & $3+$ & $6\left(O_{\mathrm{h}}\right)$ & 10.0 & 6 \\
\hline $\begin{array}{l}\mathrm{FePO}_{4} \\
\text { Synthetic }\end{array}$ & $3+$ & $4\left(T_{\mathrm{d}}\right)$ & 16.7 & 5 \\
\hline $\begin{array}{l}\mathrm{Fe}_{2} \mathrm{SiO}_{4} \\
\text { Synthetic }\end{array}$ & $2+$ & $6\left(O_{\mathrm{h}}\right)$ & 28.6 & 4 \\
\hline $\begin{array}{l}\mathrm{Fe}_{1.5} \mathrm{Mg}_{0.5} \mathrm{Al}_{9.1} \mathrm{Si}_{3.9}(\mathrm{OH})_{2} \\
\text { Staurolite: Georgia (USA) }\end{array}$ & $2+$ & $4\left(T_{\mathrm{d}}\right)$ & 3.7 & 8 \\
\hline
\end{tabular}

were confirmed to be single phase by powder X-ray diffraction. The expected $\mathrm{Fe}$ speciation was verified by ${ }^{57} \mathrm{Fe}$ Mössbauer analysis.

\subsection{X-ray absorption spectroscopy}

Fe K-edge XANES data were acquired in transmission mode using a commercially available laboratory spectrometer and the DUBBLE beamline, BM 26, of the European Synchrotron Radiation Facility.

The laboratory spectrometer, based on the design of Seidler and co-workers (Seidler et al. 2014, 2016; Mortensen et al. 2016; Jahrman et al. 2019), was an EasyXAFS XES100 system, equipped with an air cooled $\mathrm{X}$-ray tube operating at $25 \mathrm{kV}$ and $4 \mathrm{~mA}$, with a Hitachi Vortex Silicon Drift Detector (SDD). The spectrometer was configured with a Ge (620) SBCA to scan the energy range $6970-7340 \mathrm{eV}$, with a step size of $0.25-1.00 \mathrm{eV}$ and constant count time of $4 \mathrm{~s} /$ point in the XANES region (total scan time $30 \mathrm{~min}$. for $0.25 \mathrm{eV}$ step size). The energy resolution of the SDD is $c .140 \mathrm{eV}$, enabling rejection of the harmonic content of the incident beam and background scatter. A He-flight path was used to minimise air scatter and absorption. Transmission data were acquired with $\left(I_{t}(E)\right)$ and without the sample $\left(I_{0}(E)\right)$, using the same scan parameters. The Ge (620) monochromator was aligned using the clock angle procedure of Mortensen and Seidler (2017), to account for the crystal miscut.

The DUBBLE beamline is situated on a bending magnet and was configured with a Si (111) channel cut monochromator and $\mathrm{Si}$ mirror for harmonic rejection (Borsboom et al. 1988). Incident and transmitted beam intensities were measured using ionization chambers, filled with mixtures of $\mathrm{He}$ and $\mathrm{N}_{2}$, operated in a stable region of their $I / V$ curve. For synchrotron data, a step size of $0.25 \mathrm{eV}$ was used in the XANES region with a count time of $1 \mathrm{~s} /$ point.

Samples were prepared from finely ground specimens dispersed in $70 \mathrm{mg}$ of polyethylene glycol, pressed into $13 \mathrm{~mm}$ diameter pellets, to achieve a thickness of one absorption length. Data reduction and analysis was performed using the programs Athena and Hephaestus (Ravel et al. 2005). Calibration of the energy scale was achieved by reference to a $5 \mu \mathrm{m}$ Fe foil (Goodfellow Cambridge Ltd.), for which the first peak in the derivative spectrum was set to $7112.00 \mathrm{eV}$, as defined by Bearden and Burr (1967) for the Fe K absorption edge. For laboratory XANES data, Fe foil data were acquired using the same methods as for the sample materials. The Bragg angle of $\theta=77.7084^{\circ}$, corresponding to the first peak of the derivative spectrum, was set equivalent to $7112.00 \mathrm{eV}$, enabling the energy scale to be calibrated according to:

$$
E_{c}=\frac{E_{m} \sin (\theta)}{\sin (\theta+\Delta \theta)}
$$

where $E_{\mathrm{c}}$ the calibrated energy, $E_{\mathrm{m}}$ is the measured energy, and $\Delta \theta$ is the difference between the Bragg angle corresponding to $E_{\mathrm{c}}$ and $E_{\mathrm{m}}$.

For synchrotron XANES data, an Fe foil was measured simultaneously with the samples, and the energy scale calibrated using the alignment tool in the Athena program (Ravel et al. 2005). Extraction and analysis of the preedge feature in Fe K-edge XANES data was achieved using Microsoft Excel software, to first fit a spline function to model and subtract the contribution of the edge step. The pre-edge envelope was then deconvoluted by fitting Gaussian components using a linear least-squares refinement to optimise the normalised height, full width at half height, and energy position, so as to minimise the difference between the observed and calculated envelope determined from the sum of the fitted Gaussian components. Fitting initially utilised two Gaussian components with the significance of a third component tested by inspection of the improvement in goodness of fit. The total integrated intensity and centroid energy of the pre-edge feature were determined, respectively, from the sum of the integrated intensities and intensity weighted average energy position of the Gaussian components. The same initial parameters were used for all data sets, and several sets of different initial parameters trialled, to ensure that convergence did not arise in a local minimum. This approach is a simplification of that applied by Wilke et al. 2001), which employed pseudo-Voigt components (Petit et al. 2001; Wilke et al. 2001). 


\section{Results}

\subsection{Spectrometer performance at Fe K-edge}

The robustness of the laboratory spectrometer and resolution at the $\mathrm{Fe} \mathrm{K}$-edge was investigated by acquisition and analysis of XANES data from an $\mathrm{Fe}$ foil. Figure 3 shows the result of a merging ten individual XANES scans $(0.25 \mathrm{eV}$ resolution, black line); between each individual scan, the sample carousel was removed and the Fe foil unmounted. Data were background subtracted and normalised prior to merging, but were not aligned. The resulting standard deviation in the merged data (red line in Fig. 3), attests to robustness of the spectrometer alignment and scan to scan reproducibility. Next, as shown in Fig. 4, laboratory data were acquired at step sizes of $0.25,0.50,0.75$ and $1.00 \mathrm{eV}$ in the XANES region (points) and compared with synchrotron data from the DUBBLE beamline acquired at $0.25 \mathrm{eV}$ step size (solid lines), as the first derivative to amplify the XANES features. Comparison of these spectra shows that a step size of $0.5 \mathrm{eV}$ accurately approximates the synchrotron data, but step sizes of $0.75 \mathrm{eV}$ or greater do not fully resolve features apparent in the synchrotron data. The resolution of our laboratory data are therefore comparable with that achievable with a Si (111) monochromator on a bending magnet beamline at a third generation synchrotron source, providing confidence in the characterisation of weak pre-edge features. Note that the core-hole life time is estimated to impose a limiting resolution of $1.25 \mathrm{eV}$ at the Fe K-edge (Krause and Oliver 1979), which dominates the resolution of both our laboratory and synchrotron data. For the purpose of head to head comparison a step size of $0.25 \mathrm{eV}$ was maintained for acquisition of XANES data from $\mathrm{Fe}$ reference compounds.

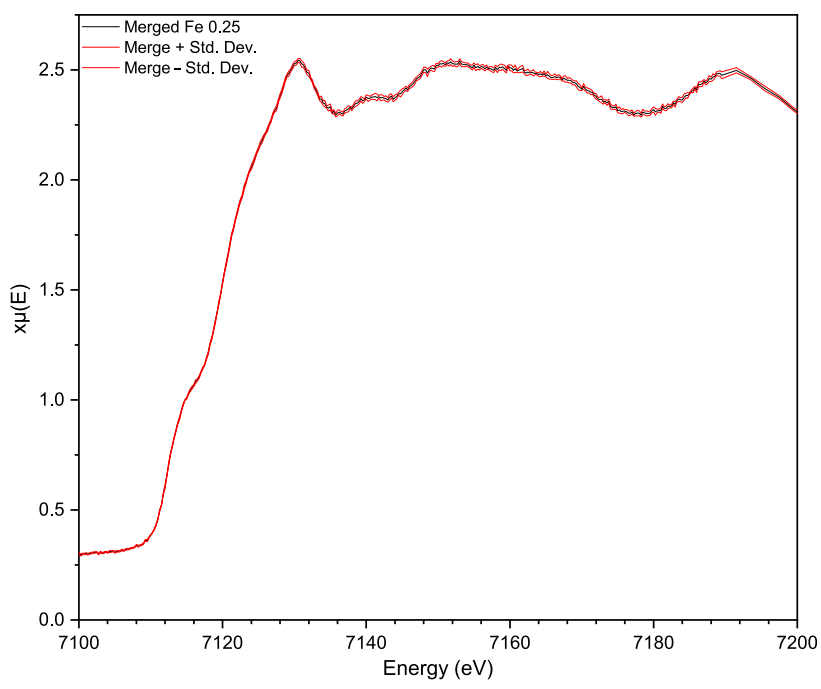

Fig. 3 Merge of ten independent XANES data sets from Fe foil $(0.25$ $\mathrm{eV}$ resolution, black line) with standard deviation (red line).

\subsection{Laboratory and synchrotron Fe K-edge XANES of model compounds}

Figure 5 compares the laboratory and synchrotron $\mathrm{Fe}$ $\mathrm{K}$-edge XANES data acquired from the reference compounds in Tab. 1. Overall, the observed chemical shift and XANES features are in excellent agreement between the data sets, when the synchrotron data are convoluted by a Lorentzian function of $0.75 \mathrm{eV}$ of full-width at halfmaximum $(F W H M)$. For the staurolite data in particular, which present relatively sharp features in the region of the white line, this convolution greatly improves the agreement between synchrotron and laboratory data. We also corrected laboratory data for the "leakage" effect, which arises from contamination of $I_{0}$ and $I_{\mathrm{t}}$ by photons arising from the low-energy tail of the monochromator function, harmonics and scattering $\left(I_{\mathrm{lk}}\right)$ (Stern and Kim

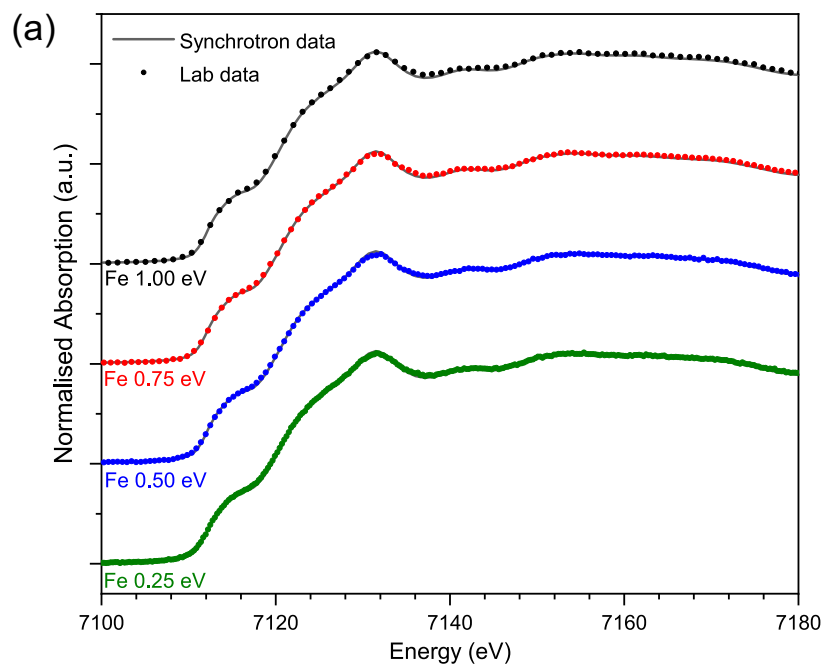

(b)

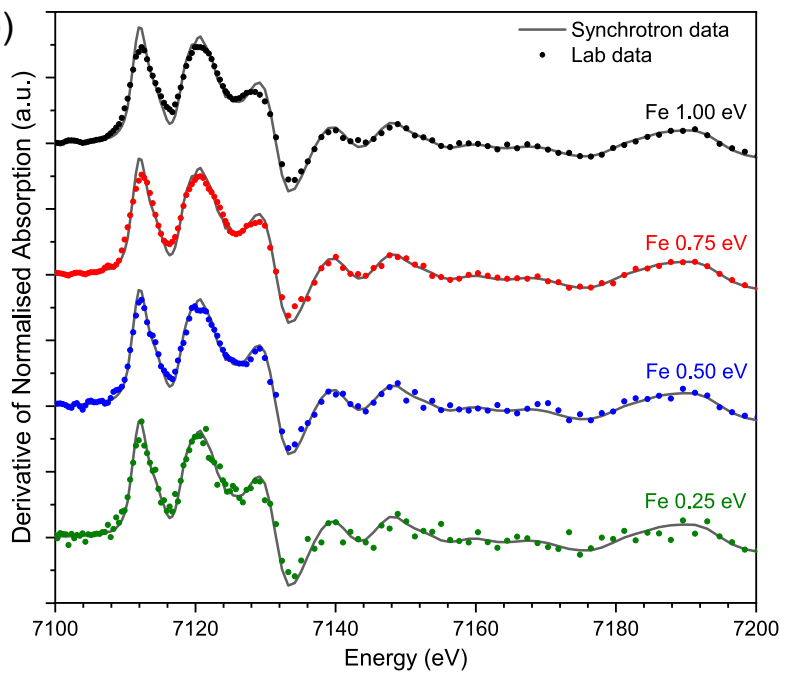

Fig. 4a - Comparison of synchrotron $(0.25 \mathrm{eV}$ resolution, black line $)$ and laboratory $(0.25-1.00 \mathrm{eV}$ resolution, points) acquired XANES data; b - Comparison of first derivative of synchrotron $(0.25 \mathrm{eV}$ resolution, black line) and laboratory (0.25-1.00 eV resolution, points) acquired XANES data, to emphasise correspondence of features. 
1981). Consequently, $\mu=\ln \left(I_{0}-I_{0,1 \mathrm{k}}\right) /\left(I_{\mathrm{t}}-I_{\mathrm{t}, \mathrm{k}}\right)$, and thus $\mu$ no longer strictly depends on $I_{0}$ and $I_{\mathrm{t}}$. In simple terms, the distortion arises because, at the white line, where $\mu$ is a maximum, $I_{\mathrm{lk}}$ makes a non-negligible contribution to the measured $I_{\mathrm{t}}$, which is small because of the dilute concentration in a relatively absorbing matrix. Thus, the measured absorption is less than the true absorption. In the present investigation, this correction had marginal impact on the laboratory XANES data. Figure 6 and Tab. 2 compare the extracted pre-edge features from laboratory and synchrotron XANES data and the modelled envelope of the Gaussian components (here the synchrotron data are not convoluted by Lorentzian broadening). For each material, the pre-edge features of the laboratory and synchrotron XANES data were adequately modelled using the same number of Gaussian components (either two or three). Although the laboratory data clearly demonstrate more scatter in comparison to the synchrotron data, as expected due to lower signal to noise ratio, the correspondence between the data sets is remarkable. The centroid energy of the pre-edge features of $\mathrm{Fe}^{2+}$ and $\mathrm{Fe}^{3+}$ species are clearly distinguished at $7112.9 \pm 0.1 \mathrm{eV}$ and $7114.5 \pm 0.1 \mathrm{eV}$ respectively, in both the laboratory and synchrotron data. For each Fe species, the measured normalised intensity of the pre-edge feature is also in excellent agreement between laboratory and synchrotron data sets. As expected, the pre-edge features of the tetrahedral Fe species present in $\mathrm{FePO}_{4}$ and $\mathrm{Fe}_{1.5} \mathrm{Mg}_{0.5} \mathrm{Al}_{9.1} \mathrm{Si}_{3.9}(\mathrm{OH})_{2}$, have considerably greater intensity than those of the octahedral species present in $\mathrm{NaFeSi}_{2} \mathrm{O}_{6}$ and $\mathrm{Fe}_{2} \mathrm{SiO}_{4}$. As noted above, and discussed below, the pre-edge features

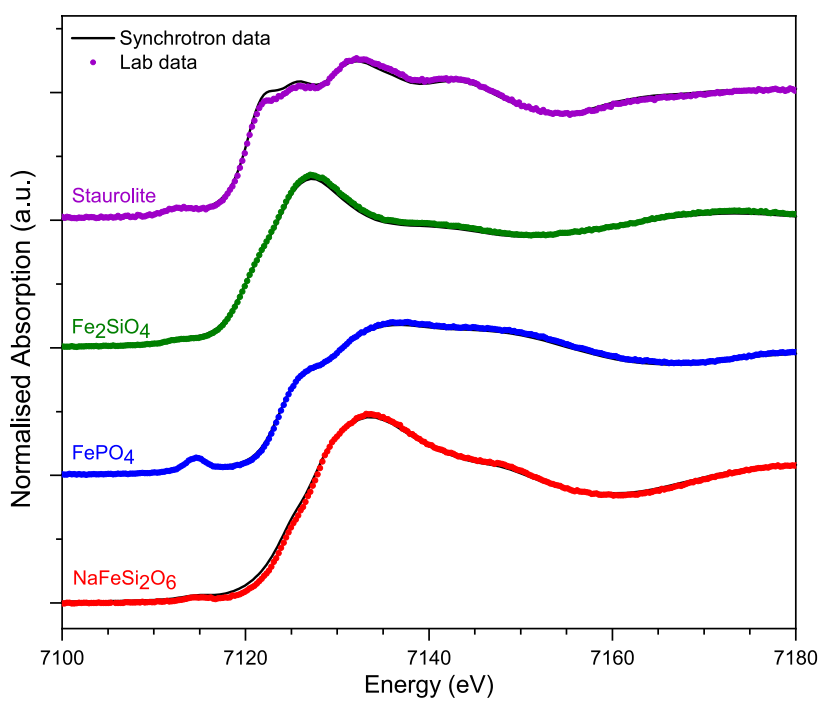

Fig. 5 Comparison of normalised transmission mode Fe K-edge XANES spectra from reference compounds $\mathrm{NaFeSi}_{2} \mathrm{O}_{6}, \mathrm{FePO}_{4}, \mathrm{Fe}_{2} \mathrm{SiO}_{4}$, and staurolite $-\mathrm{Fe}_{1.5} \mathrm{Mg}_{0.5} \mathrm{Al}_{9.1} \mathrm{Si}_{3.9}(\mathrm{OH})_{2}$, (prepared to $\mu \mathrm{x}=1$ ), acquired using laboratory XAS spectrometer (points) and DUBBLE beamline (solid line); synchrotron data were broadened by a Lorentzian function of $0.75 \mathrm{eV}$ FWHM. are primarily associated with $1 \mathrm{~s}-3 \mathrm{~d}$ quadrupole transitions, which are only weakly allowed for octahedral $\mathrm{Fe}$ since the initial and final states are centrosymmetric. In the non-centrosymmetric tetrahedral environments, the transition probability is enhanced due to admixture of unoccupied $4 p$ orbitals in the final state. Figure 7 compares the total integrated intensity and centroid energy of modelled pre-edge features extracted from our laboratory and synchrotron XANES data, and the synchrotron data reported by Petit et al. (2001) and Wilke et al. (2001). To construct this plot, the data of Wilke et al. (2001) (which were calibrated to $E_{0}=7111.08 \mathrm{eV}$ ), were shifted by $+0.92 \mathrm{eV}$, consistent with our absolute energy scale. Figure 7 shows that $\mathrm{Fe}$ speciation is clearly differentiated according to the total integrated intensity and centroid position of the pre-edge feature. The data points determined from laboratory and synchrotron data, acquired from identical materials, and corresponding data points acquired by Wilke et al. (2001), on their materials, are seen to be in good overall agreement (solid points in Fig. 7). A systematic difference of c. $0.25 \mathrm{eV}$ is apparent between the pre-edge centroid energy determined from our laboratory and synchrotron data. This is of the order of the measurement step size and, therefore, likely arises from a small difference in calibration of the absolute energy scale (see also Tab. 2). The spread in determined total integrated intensities is approximately $15-20 \%$ relative, with the laboratory determination systematically lower than our synchrotron determination. In the case of our laboratory data, the contribution of intensity in the tails of the pre-edge features is underestimated by the fitted Gaussian components, due to the lower signal to noise. Nevertheless, it is clear that both our laboratory and synchrotron data fall within the known $\mathrm{Fe}^{2+}$ and $\mathrm{Fe}^{3+}$ speciation fields, as shown by Fig. 7. Some scatter is apparent between our synchrotron data points and those of Wilke et al. (2001), for the same nominal materials. However, given that the samples, experimental set ups, and data analysis methods are not identical, we believe the overall agreement between the studies to validate the determination of Fe speciation by laboratory XANES data.

\section{Discussion}

The data presented in Figs 5-7 show that good quality XANES data may be acquired in a matter of hours from compounds with moderately low to high Fe concentrations, using a commercially available spectrometer, enabling routine speciation of $\mathrm{Fe}$ in the laboratory. Optimisation of the data acquisition strategy may yield efficiency gains for routine application. Good signal to noise ratio, and a sufficiently small step size, are essential 
in the region of the pre-edge and rising absorption edge, to enable accurate isolation of the pre-edge feature with optimal resolution. However, outside of this range, the density of data points used in this study could potentially be reduced by a factor of 2-4, without impacting on accuracy or precision of the pre-edge analysis, for high throughput routine analysis.

The weak pre-edge features observed in Fe K-edge XANES data are associated primarily with $1 \mathrm{~s}-3 \mathrm{~d}$ (quadrupole) and $1 \mathrm{~s}-4 \mathrm{p}$ (dipole) electronic transitions (Waychunas et al. 1983; Bajt et al. 1994; Galoisy et al. 2001; Petit et al. 2001; Wilke et al. 2001; Berry et al. 2003; Joseph et al. 2017). The pre-edge envelope is typically comprised of two or more components that arise from transitions to $3 \mathrm{~d}$ final states. They are non-degenerate as a result of the crystal field imposed by the local environment of the Fe absorber. Thus, crystal field theory predicts two and three allowed transitions, respectively, for $\mathrm{Fe}^{2+}\left([\mathrm{Ar}] 3 \mathrm{~d}^{6} h s\right)$ in tetrahedral and octahedral field, whereas, two allowed transitions are predicted for $\mathrm{Fe}^{3+}$ ([Ar] $\left.3 \mathrm{~d}^{5} h s\right)$ in tetrahedral and octahedral crystal fields respectively ( $h s$ : high spin) (Westre et al. 1997; Baker et al. 2017). Additional contributions to the pre-edge feature have been reported for some distorted $\mathrm{Fe}^{3+}$ octahedral environments (such as in $\mathrm{Fe}_{2} \mathrm{O}_{3}$ ) which are attributed to admixture of $4 p$ states and availability of unoccupied delocalised states (Dräger et al. 1988; de Groot et al. 2009). In this investigation, and others (Petit et al. 2001; Wilke et al. 2001), deconvolution of the pre-edge feature requires fewer components than the number of allowed transitions, due to a combination of the magnitude of the crystal field splitting, distortion from ideal co-ordination geometry, limited spectral resolution compared to the core-hole life time, and other factors. Nevertheless, as demonstrated by Fig. 7, the total integrated intensity and centroid energy of the pre-edge feature can be accurately estimated by fitting of Gaussian or pseudo-Voigt functions, and is correlated with the electronic configuration and symmetry of the absorber and hence oxidation state and co-ordination number.

The chemical shift between the centroid energy of $\mathrm{Fe}^{2+}$ and $\mathrm{Fe}^{3+}$ species determined in this investigation was $1.6 \pm 0.1 \mathrm{eV}$ for both laboratory and synchrotron XANES data, in excellent agreement with the value of $1.4 \pm 0.1$
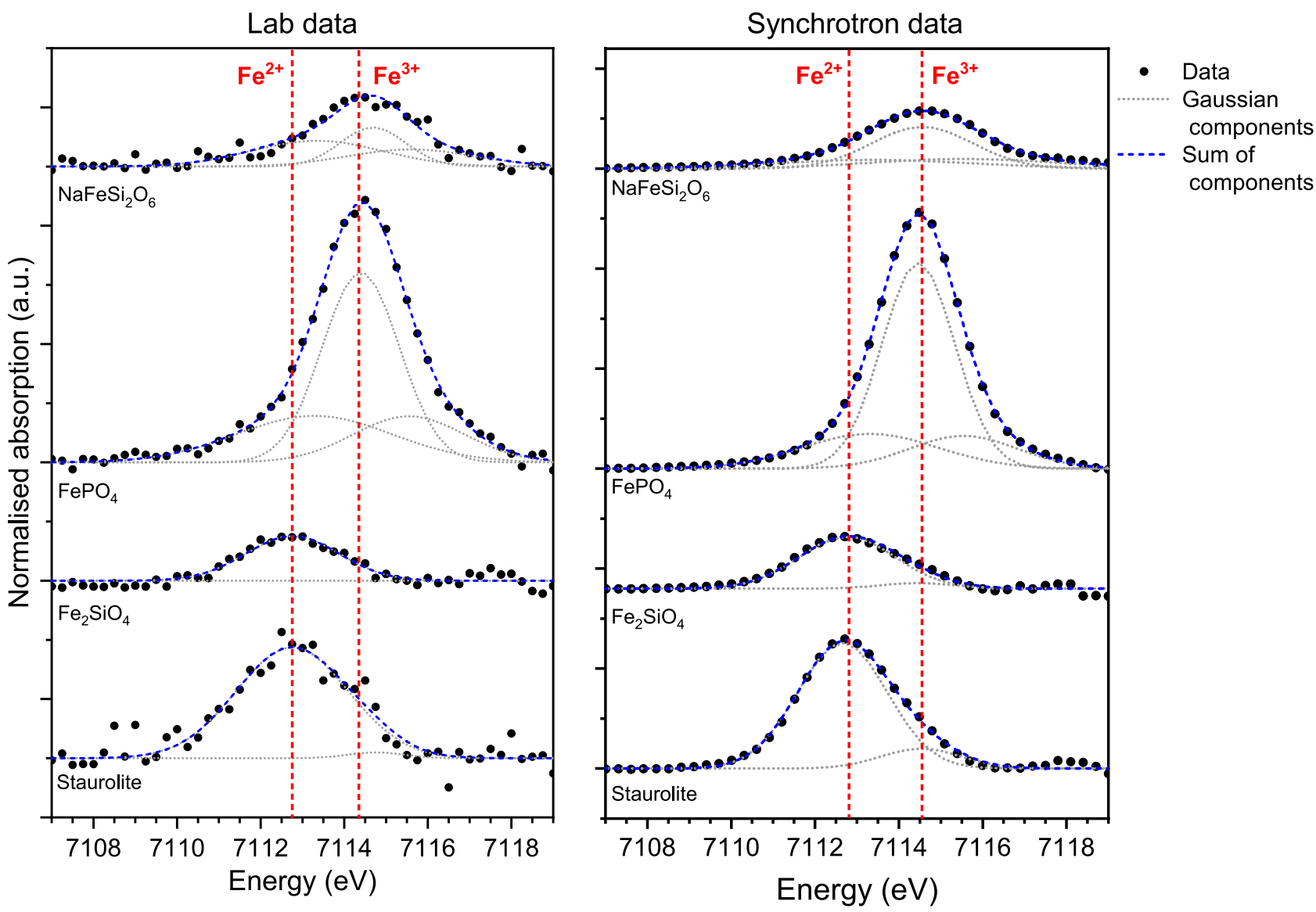

Fig. 6 Normalised pre-edge spectra at Fe K-edge (points) from reference compounds $\mathrm{NaFeSi}_{2} \mathrm{O}_{6}, \mathrm{FePO}_{4}, \mathrm{Fe}_{2} \mathrm{SiO}_{4}$, and staurolite $\left[\mathrm{Fe}_{1.5} \mathrm{Mg}_{0.5}\right.$ $\left.\mathrm{Al}_{9.1} \mathrm{Si}_{3.9}(\mathrm{OH})_{2}\right]$, acquired using laboratory XAS spectrometer and DUBBLE beamline, showing fitted Gaussian components (grey dotted line) and their summation envelope (blue dotted line). 
Tab. 2 Integrated intensity and centroid energy for Gaussian components fitted to pre-edge features, and intensity-weighted centroid position and total integrated intensity of fitted pre-edge envelope

\begin{tabular}{|c|c|c|c|c|c|c|c|c|}
\hline \multirow[b]{2}{*}{ Material } & \multicolumn{2}{|c|}{ Gaussian 1} & \multicolumn{2}{|c|}{ Gaussian 2} & \multicolumn{2}{|c|}{ Gaussian 3} & \multirow[b]{2}{*}{$\begin{array}{c}\text { Centroid energy } \\
(\mathrm{eV})\end{array}$} & \multirow[b]{2}{*}{$\begin{array}{l}\text { Total integrated intensity } \\
\text { (a.u). }\end{array}$} \\
\hline & $\begin{array}{l}\text { Energy } \\
(\mathrm{eV})\end{array}$ & $\begin{array}{l}\text { Intensity } \\
\text { (a.u.) }\end{array}$ & $\begin{array}{c}\text { Energy } \\
(\mathrm{eV})\end{array}$ & $\begin{array}{l}\text { Intensity } \\
\text { (a.u.) }\end{array}$ & $\begin{array}{c}\text { Energy } \\
(\mathrm{eV})\end{array}$ & $\begin{array}{l}\text { Intensity } \\
\text { (a.u.) }\end{array}$ & & \\
\hline $\mathrm{NaFe}_{2} \mathrm{SiO}_{6}$ & 7113.3 & 0.042 & 7114.7 & 0.032 & 7115.6 & 0.027 & 7114.5 & 0.102 \\
\hline Aegerine: Langesund Fjord (Norway) & 7113.4 & 0.027 & 7114.6 & 0.063 & 7115.6 & 0.028 & 7114.6 & 0.117 \\
\hline $\mathrm{FePO}_{4}$ & 7113.3 & 0.091 & 7114.5 & 0.186 & 7115.4 & 0.065 & 7114.4 & 0.343 \\
\hline Synthetic & 7113.3 & 0.071 & 7114.5 & 0.225 & 7115.5 & 0.053 & 7114.4 & 0.349 \\
\hline $\mathrm{Fe}_{2} \mathrm{SiO}_{4}$ & 7112.7 & 0.063 & 7115.8 & 0.002 & & & 7112.8 & 0.063 \\
\hline Synthetic & 7112.8 & 0.075 & 7114.5 & 0.006 & & - & 7112.9 & 0.081 \\
\hline $\mathrm{Fe}_{1.5} \mathrm{Mg}_{0.5} \mathrm{Al}_{9.1} \mathrm{Si}_{3.9}(\mathrm{OH})_{2}$ & 7112.8 & 0.157 & 7114.8 & 0.004 & & & 7112.9 & 0.161 \\
\hline Staurolite: Georgia (USA) & 7112.7 & 0.169 & 7114.5 & 0.024 & - & - & 7112.9 & 0.192 \\
\hline
\end{tabular}

Values in bold type are derived from laboratory data; those in normal type from synchrotron data

eV determined by Wilke et al. (2001). Our laboratory and synchrotron data can be brought into approximate co-incidence in Fig. 7, by a shift of approximately 0.25 $\mathrm{eV}$ in energy and 0.025 a.u. in total integrated intensity, which implies that the differences between our measurements arise principally from systematic error. Therefore, a comprehensive reference data set (including reference mixtures) should be acquired to define the speciation fields (and their joins), for each specific instrument (whether laboratory or synchrotron) to enable the most accurate speciation. This will form the objective of the next phase of this study.

As pointed out by Wilke et al. (2001), Fe speciation can be determined unambiguously in the case of a single dominant species or a binary mixture, from analysis of the pre-edge feature in Fe K-edge XANES. However, unique determination of more complex mixtures, or the presence of 5 coordinate $\mathrm{Fe}$, is more difficult to ascertain, although the location of extracted data in the structure field map of Fig. 7 at least provides some insight into the likely component species. For such cases, modelling of the extended X-ray absorption fine structure, additional ${ }^{57} \mathrm{Fe}$ Mössbauer data, and/or simulation of XANES spectra, are required to give a unique determination.

\section{Conclusions}

We have successfully validated the methodology of Wilke and co-workers (Petit et al. 2001; Wilke et al. 2001), for analysis of weak pre-edge features in Fe K-edge XANES for determination of Fe speciation, using data acquired from model compounds in a matter of hours on a commercially available laboratory X-ray spectrometer. Quantitative agreement was observed between the total integrated intensity and centroid energy of the pre-edge features determined from laboratory and synchrotron data, and with previously published independent data. The primary sources of discrepancy in these parameters within our study were systematic error, arising from marginal difference in calibration of the absolute energy scale and under- estimation of total integrated intensity due to lower signal to noise ratio in the laboratory data. The latter could be compensated, to an extent, by increased effective counting time. Therefore, for accurate speciation, data from a library of reference compounds should be established for the particular laboratory or synchrotron instrument. This proof of concept study has also clearly established the feasibility of data acquisition and analysis for relatively dilute $\mathrm{Fe}$ absorber concentrations. The rate of core-hole generation in our set up is not favourable for fluorescence data acquisition as a result of the low-power $100 \mathrm{~W}$ X-ray source; however, successful laboratory fluorescence XAS measurements using a $1.5 \mathrm{~kW}$ X-ray source were recently reported by Honkanen et al. (2019). Nevertheless, as we have demonstrated here, transmission mode XANES may

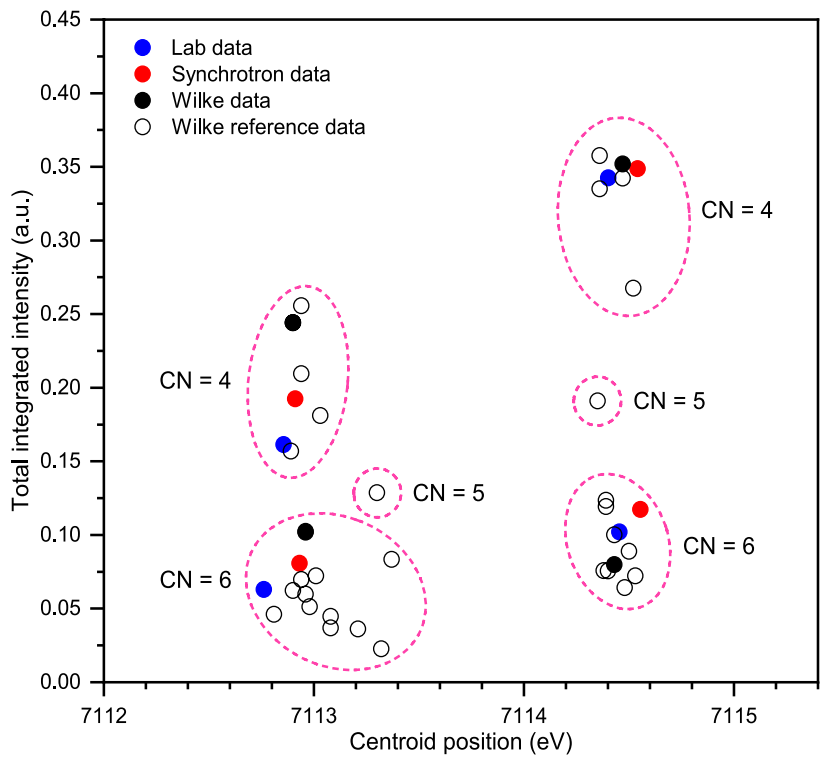

Fig. 7 Speciation field diagram, based on total integrated intensity and centroid energy of pre-edge feature of Fe K-edge XANES data. Data points derived from analysis of our laboratory and synchrotron data are shown as solid blue and red circles, respectively, equivalent data points from Wilke et al. (2001) as solid black circles. Other reference data from Wilke et al. (2001) are shown as open black circles. Note that data from Wilke et al. (2001), are shifted by $+0.92 \mathrm{eV}$ consistent with our absolute energy scale as described in the text. 
be acquired from relatively dilute absorber concentrations without heroic data acquisition times, in favourable circumstances. Indeed, we have recently reported further exploitation of this approach to speciation of $\mathrm{Ce}$ and $\mathrm{U}$ $\mathrm{L}_{3}$ edge XANES, with absorber concentrations of only a few mol. \% (Mottram et al. 2020).

Acknowledgements. We are grateful for financial support from the Nuclear Decommissioning Authority and EPSRC under grant numbers EP/M026566/1, EP/S01019X/1, EP/ N017870/1 and EP/R511754/1. This research utilised the HADES/MIDAS facility at The University of Sheffield established with financial support from EPSRC and BEIS, under grant EP/T011424/1. This research was undertaken, in part, at the DUBBLE beamline, BM 26, at the European Synchrotron Radiation Facility. We gratefully acknowledge G.T. Seidler and D.R. Mortensen for useful discussions in support of this study. We are grateful to Dr Jakub Plášil for facilitating publication of this manuscript as the handling editor and to the anonymous reviewers for their careful consideration and recommendations.

\section{References}

ARnOLD H (1986) Crystal structure of $\mathrm{FePO}_{4}$ at 294 and 20 K. Z Kristallogr 177: 139-142

BaJt S, SUtTon SR, DeLANEY JS (1994) X-ray microprobe analysis of iron oxidation states in silicates and oxides using X-ray absorption near edge structure (XANES). Geochim Cosmochim Acta 58: 5209-5214

Baker ML, Mara MW, Yan JJ, Hodgson KO, Hedman B, Solomon EI (2017) K- and L-edge X-ray Absorption Spectroscopy (XAS) and Resonant Inelastic X-ray Scattering (RIXS) determination of Differential Orbital Covalency (DOC) of transition metal sites. Coord Chem Rev 345: 182-208

Baum E, Treutmann W, Behruzi M, Lottermoser W, Amthauer G (1988) Structural and magnetic properties of the clinopyroxenes $\mathrm{NaFeSi}_{2} \mathrm{O}_{6}$ and $\mathrm{LiFeSi}_{2} \mathrm{O}_{6}$. Z Kristallogr 183: 273-284

BEARDEN JA, BURr AF (1967) Reevaluation of X-ray atomic energy levels. Rev Mod Phys 39: 125

Berry AJ, O’Neill hS, Jayasuriya KD, Campbell SJ, ForAN GJ (2003) XANES calibrations for the oxidation state of iron in a silicate glass. Amer Miner 88: 967-977

Blachucki W, Czapla-Masztafiak J, Sa J, Szlachetko J (2019) A laboratory-based double X-ray spectrometer for simultaneous X-ray emission and X-ray absorption studies. J Anal At Spectrom 34: 1409-1415

Borsboom M, Bras W, Cerjak I, Detollenaere D, Glastra Van Loon D, Goedtkindt P, Konijneburg M, Lassing P, Levine YK, Munneke B, Oversluizen M,
Van Tol R, Vlieg E (1988) The Dutch-Belgian beamline at the ESRF. J Synchroton Radiat 5: 518-520

De Groot F, Vanko G, Glatzel P (2009) The 1s X-ray absorption pre-edge structures in transition metal oxides. J Phys Condens Matter: 21104207

DräGer G, Frahm R, Materlik G, BrüMmer O (1988) On the multipole character of the X-ray transitions in the pre-edge structure of Fe K absorption spectra. An experimental study. Phys Status Solidi B 146: 287-294

Galoisy L, Calas G, ARrio MA (2001) High-resolution XANES spectra of iron in minerals and glasses: structural information from the pre-edge region. Chem Geol 174: $307-319$

Greenwood N, Earnshaw A (1997) Chemistry of the Elements, $2^{\text {nd }}$ Edition. Butterworth-Heinemann, Oxford, pp 1-1342

Hawthorne FC, Ungaretti L, Oberti R, Caucia A, CALlegari A (1993) The crystal chemistry of staurolite; 1. Crystal structure and site populations. Canad Mineral 31: $551-582$

Holden WM, Hoidn OR, Ditter AS, Seidler GT, Kas J, Stein JL, Cossairt BM, Kosimor SA, Guo JH, Ye YF, Marcus MA, FAKRa S (2017) A compact dispersive refocusing Rowland circle X-ray emission spectrometer for laboratory, synchrotron, and XFEL applications. Rev Sci Instrum 88: 073904

Honkanen AP, Ollikkala S, Ahopelto T, Kallio AJ, BLOMBERG M, HUOTARI S (2019) Johann-type laboratoryscale X-ray absorption spectrometer with versatile detection modes. Rev Sci Instrum 90: 033107

Jahrman EP, Holden WM, Ditter AS, Mortensen DR, Seidler GT, Fister TT, Kozimor SA, Piper LFJ, Rana J, Hyatt NC, Stennett M (2019) An improved laboratory-based X-ray absorption fine structure and $\mathrm{X}$-ray emission spectrometer for analytical applications in materials chemistry research. Rev Sci Instrum 90: 024106

Joseph K, Stennett MC, Hyatt NC, Asuvathraman R, Dube CL, Gandy AS, Kutty KVG, Jolley K, Rao PRV, SмIтн R (2017) Iron phosphate glasses: bulk properties and atomic scale structure. J Nucl Mater 494: 342-353

Krause MO, Oliver JH (1979) Natural widths of atomic $\mathrm{K}$ and $\mathrm{L}$ levels, $\mathrm{K}_{\alpha} \mathrm{X}$-ray lines and several KLL Auger lines. J Phys Chem Ref Data 8: 320-338

Malzer W, Grotzsch G, Gnewkow R, Schlesiger C, Kowalewski F, Van Kuiken B, Debeer S, KanNGiesser B (2018) A laboratory spectrometer for high throughput $\mathrm{X}$-ray emission spectroscopy in catalysis research. Rev Sci Instrum 89: 113111

MoRTENSEN DR, SEIDLER GT (2017) Robust optic alignment in a tilt-free implementation of the Rowland circle spectrometer. J Electron Spectrosc Relat Phenom 215: 8-15

Mortensen DR, Seidler GT, Ditter AS, Glatzel P (2016) Benchtop nonresonant $\mathrm{X}$-ray emission spectroscopy: 
coming soon to laboratories and XAS beamlines near you? J Phys Conf Ser 712: 012036

Mottram LM, Dixon Wilkins MC, Blackburn LR, OUlton T, Stennett MC, Sun SK, Corkhill CL, Hyatt NC (2020) A feasibility investigation of laboratory based X-ray absorption spectroscopy in support of nuclear waste management. MRS Adv 5: 27-35

Nemeth Z, Szlachetko J, Bajnoczi EG, Vanko G (2016) Laboratory von Hamos X-ray spectroscopy for routine sample characterization. Rev Sci Instrum 87: 103105

Petit PE, Farges F, Wilke M, Solé VA (2001) Determination of the iron oxidation state in Earth materials using XANES pre-edge information. J Synchroton Radiat 8: 952-954

Ravel B, Newville M (2005) ATHENA, ARTEMis, HEPHAESTUS: data analysis for X-ray absorption spectroscopy using IFEFFIT. J Synchroton Radiat 12: 537-541

Schlesiger C, Anklamm L, Stiel H, Malzer W, KanngiESSER B (2015) XAFS spectroscopy by an X-ray tube based spectrometer using a novel type of HOPG mosaic crystal and optimized image processing. J Anal At Spectrom 30: 1080-1085

Seidler GT, Mortensen DR, Remesnik AJ, Pacold JI, BALl NA, BARry N, Styczinski M, HoIdN OR (2014) A laboratory-based hard X-ray monochromator for high-resolution X-ray emission spectroscopy and X-ray absorption near edge structure measurements. Rev Sci Instrum 85: 113906
Seidler GT, Ditter AS, BAll NE, Remesnik AJ (2016) A modern laboratory XAFS cookbook. J Phys Conf Ser 712: 012015

SMYTH JR (1975) High temperature crystal chemistry of fayalite. Amer Miner 60: 1092-1097

Stern EA, Kim K (1981) Thickness effect on the extendedX-ray-absorption-fine-structure amplitude. Phys Rev B 23: 3781-3787

TAYLOR KG, KonHAuser KO (2011) Iron in Earth surface systems: a major player in chemical and biological processes. Elements 7: 83-87

Waychunas GA, Apted MJ, Brown GE (1983) X-ray K-edge absorption-spectra of Fe minerals and model compounds - near-edge structure. Phys Chem Miner 10: 1-9

Westre TE, Kennepoh P, Dewitt JG, Hedman B, HodGSON KO, Solomon EI (1997) A Multiplet analysis of Fe $\mathrm{K}$-edge $1 \mathrm{~s} \rightarrow 3 \mathrm{~d}$ pre-edge features of iron complexes. J Amer Chem Soc 119: 6297-6314

Wilke M, Farges F, Pettit PE, Brown GE, Martin F (2001) Oxidation state and coordination of Fe in minerals: an Fe K-XANES spectroscopic study. Amer Miner 86: 714-730

Wood BJ, Virgo D (1989) Upper mantle oxidation state: ferric iron contents of lherzolite spinels by ${ }^{57} \mathrm{Fe}$ Mössbauer spectroscopy and resultant oxygen fugacities. Geochim Cosmochim Acta 53: 1277-1291

Zeeshan F, Hoszowska J, Loperetti-Tornay L, Dousse JC (2019) In-house setup for laboratory-based X-ray absorption fine structure spectroscopy measurements. Rev Sci Instrum 90: 073105 